\title{
Historia ambiental del comanejo adaptativo en dos regiones pesqueras del noroeste mexicano
}

\author{
Environmental History of Adaptive Co-management \\ in Two Fishing Regions in Northwestern Mexico
}

Pablo Álvarez

UNIVERSIDAD AUTÓNOMA DE BAJA CALIFORNIA, pabloam@uabc.edu.mx

\section{Claudia Delgado}

ESCUELA DE ANTROPOLOGÍA E HISTORIA DEL NORTE DE MÉXICO, claudia_delgado@inah.gob.mx

\section{Georges Seingier}

UNIVERSIDAD AUTÓNOMA DE BAJA CALIFORNIA, FACULTAD DE CIENCIAS MARINAS, georges@uabc.edu.mx

\section{Ileana Espejel}

UNIVERSIDAD AUTÓNOMA DE BAJA CALIFORNIA, ileana.espejel@uabc.edu.mx

La península de Baja California es un territorio aislado destacable por varios casos de pesquerías de exportación y su organización social. En el presente escrito se describe la historia ambiental de dos sistemas de manejo pesquero (abulón/langosta y erizo) en la costa del Pacífico. El comienzo de ambos sistemas es equivalente en el manejo intensivo de pesquerías con alto valor comercial en Asia y la conversión de trabajadores en tierra (rancheros y ejidatarios) a pescadores. Un segundo momento es la organización de los pescadores en cooperativas pesqueras donde comienza el comanejo de las pesquerías de abulón, langosta en el sur y erizo rojo en el norte. Como respuesta a los cambios propios de la naturale$\mathrm{za}$, de las organizaciones sociales, institucionales y comerciales y ahora al cambio climático, los pescadores han desarrollado esquemas de comanejo adaptativo que proveen experiencias nacionales exitosas para inspirar esquemas de manejo sostenible en otras pesquerías.

Palabras clave: océano Pacífico, pesquerías, desarrollo, cooperativa, historia ambiental.

The Baja California Peninsula is an isolated territory distinguished by the presence of export-oriented fisheries and the social organization of fishermen. The environmental histories of two fishing management schemes on the Pacific coast (abalone/lobster and sea urchin) are described. The origins of these two systems are similar in terms of (1) intensive fishing management schemes for catches that enjoy high commercial value in Asia; and (2) a change in occupations as land-based ranchers and farmers were converted into fishermen. In a second stage, fishermen organized in cooperatives, which led to the introduction of a co-management scheme to exploit abalone and lobster in the southern area and red sea urchins in the north. Responding to changes in the natural environment, social organization, institutions, commercial trends and, more recently, the climate, these fishers 
developed an adaptive co-management scheme that provides cases of successful experiences in the country that could stimulate the implementation of sustainable management schemes in other fisheries.

KeYwORDs: Pacific Ocean, fisheries, development, cooperatives, environmental history.

Fecha de recepción: 22 de noviembre de 2016 / Fecha de aprobación: 14 de junio de 2017 / Fecha de recepción de la versión final: 30 de julio de 2017

\section{INTRODUCCIÓN}

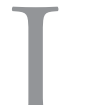

a pesca es la actividad económica más extensa en los litorales mexicanos, México se ubica entre los líderes de captura pesquera. Una historia de 40 años de explotación intensiva en las pesquerías de pequeña escala ha resultado en una sobreexplotación, al igual que las pesquerías de manera generalizada alrededor del mundo (Fulton et al., 2001; Espinoza et al., 2015). En respuesta a esta problemática mundial se han propuesto esquemas alternativos de aprovechamiento de los recursos pesqueros, uno de estos esquemas es el comanejo. Algunos casos documentados sobre comanejo son: diversas tribus autóctonas del estado de Washington (Pinkerton 2007), la estrategia de manejo del Parque Natural La Gran Barrera de Arrecife en Australia (Olsson et al., 2008), las pesquería de arrecife en Islas Vírgenes en el oriente del Mar Caribe (Carr y Heyman 2012), la pesquería de erizo de mar en Galicia (FernándezBoán et al., 2012), las pesquerías en Indonesia (Muawanah et al., 2012) y especialmente desde hace muchos años en Noruega y otros países del norte (Jentoft y McCay 1995), la cual ha sido descrita de una forma muy completa pero también crítica por Jentoft (1989) y Jentoft (2000 a y b).

El objeto de estudio del presente trabajo es el comanejo adaptativo, el cual es un concepto que retoma los aspectos del comanejo relacionados con la participación de todos los usuarios vinculados a uno o más recursos naturales en la toma de decisiones asociados a éstos y de la vinculación de estos usuarios con el manejo gubernamental de los recursos. La dimensión adaptativa, que acompaña al comanejo, se refiere al aprendizaje que los usuarios de los recursos naturales van construyendo e incorporando en sus esquemas de ma- 
nejo, para enfrentar y resolver problemas asociados a la incertidumbre, tanto de los propios recursos como, por ejemplo, del mercado. En el mejor de los casos, estos aprendizajes pueden cristalizarse mejorando y afinando las políticas correspondientes al sector y a los recursos específicos (Armitage et al., 2007).

Los sistemas de comanejo adaptativo son sistemas comunitarios flexibles (down-top) de manejo de recursos, que se desarrollan en lugares y situaciones específicas y son apoyados por organizaciones con las que trabajan de manera conjunta. El comanejo adaptativo se construye a través de la colaboración entre diversos actores y usuarios que funcionan o tienen injerencia en distintos niveles, mediante redes de usuarios locales y municipales, organizaciones regionales y nacionales e incluso algunos internacionales. En este sentido, la gobernanza se ha convertido en una alternativa de colaboración, asociación y conformación de redes distintas del control gubernamental vertical arriba-abajo (top-down). También es importante como proceso adaptativo del manejo, el capital social que se origina y fortalece a través del manejo colaborativo (Plummer y FitzGibbon $2007,42)$. En resumen, son diversos los procesos y las instituciones que resultan y evolucionan a partir del comanejo adaptativo y todas tienen un papel específico en la concreción del objetivo central de cualquier esquema o modelo de manejo, a saber la promoción de la sustentabilidad y la resiliencia (en el ecosistema, en las instituciones de manejo, en la estructura económica y sus comunidades) necesarias en un sistema saludable de recursos a través de políticas y prácticas óptimas para su manejo (Charles 2007).

Es posible analizar el desarrollo de un esquema de comanejo adaptativo mediante un enfoque histórico ambiental, ya que permite revelar los eventos históricos que surgen de las interacciones entre los sistemas social y natural (Butzer 1971; Miller 2007; Rucinque y Velásquez 2007; Hughes 2008; Endfield 2009, 223; Gallini 2009); también permite identificar las principales posibilidades y modalidades de relación entre ambos sistemas (Sauer 1938 35, 43-44; Dedina 1996; Castro 2002, 84-99; Castree et al., 2009, 1-15; Castro 2010); así como realizar un análisis contextual para identificar factores internos y externos (Edwards y Steins 1999; Espinoza-Tenorio 
et al., 2010). Además, el enfoque histórico ambiental se basa en un amplio diálogo entre disciplinas que constantemente propone perspectivas teóricas renovadas y metodologías comprensivas (Carey 2009; Pawson y Dovers 2003), ya que las dinámicas socioambientales difícilmente pueden ser explicadas con base en un enfoque monodisciplinar.

Además, la mayoría de los estudios sobre historia ambiental se han centrado en explicar las historias de los impactos negativos en el ambiente y desigualdad en el acceso a los recursos naturales (Bourassa y Strong 2000, 156; McNeill 2000; Sáenz-Arroyo et al., 2005; Hornborg 2007, 1; Jorgenson y Rice 2007, 273-274; Carey 2009; Roberts 2009; Weisz 2007, 290; Wilk 2007, 180). Esto se explica por la motivación de los investigadores por reportar y proponer soluciones a la crisis ecológica de la actualidad (Carey 2009) y para entender las relaciones entre las regiones periféricas y los países en el sentido de adaptar y mejorar estrategias de desarrollo (Barton 2006, 367).

Por lo anterior, el objetivo del presente artículo es analizar dos casos de comanejo adaptativo en dos pesquerías en el noroeste de México: el primero de ellos la región conocida como "La Pacífico Norte", ubicada en las costas occidentales de la porción central de la península bajacaliforniana y, el segundo, el "Sistema Producto Erizo”, que se ubica en costas de Ensenada, Baja California ${ }^{1}$ (figura 1).

Para poder entender el proceso y las condiciones que han permitido el desarrollo de un modelo de comanejo adaptativo en ambos casos, es necesario conocer y comprender sus respectivas historias ambientales, ya que el comanejo adaptativo no se gesta de manera espontánea, sino que se va construyendo en la vida cotidiana y coyuntural de las comunidades, usuarios e instancias gubernamentales.

${ }^{1}$ Las poblaciones de erizo rojo (Strongylocentrotus franciscanus) se encuentran en las costas del Pacífico en Canadá, Estados Unidos y el estado de Baja California en México. Tienen un alto valor economico, especialmente para su exportación a Asia. 


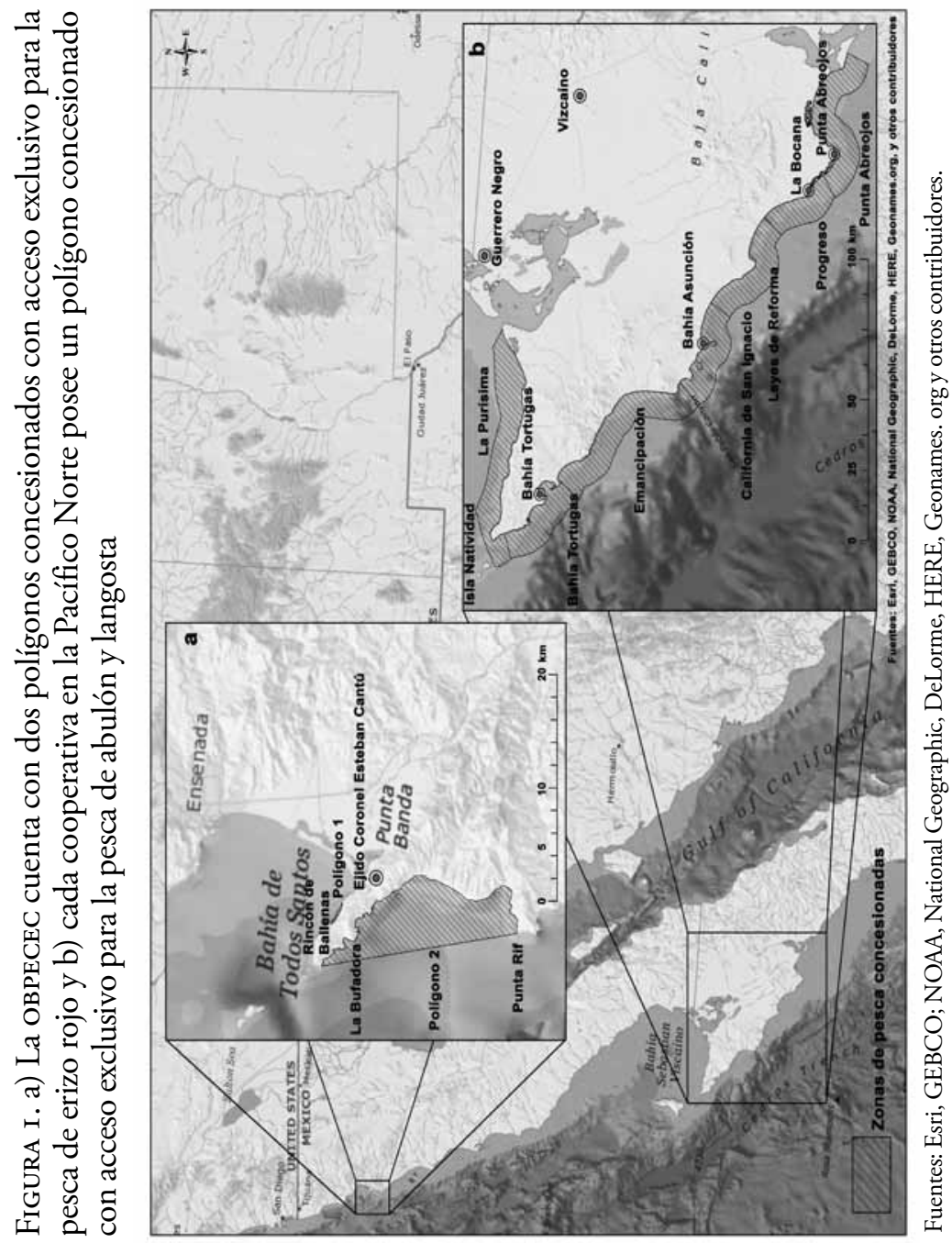




\section{MÉtodos}

Se eligieron dos casos de estudio en dos regiones de las 11 regiones pesqueras identificadas por Díaz-Uribe et al. (2013) del noroeste de México (regiones I: Ensenada, San Quintín y El Rosario y III: Pacífico Norte). Estos casos se eligieron porque, aunque no son los únicos, actualmente ambos muestran ser exitosos en el manejo y acceso a los recursos naturales (Ponce-Díaz et al., 1998; Cariño y Monteforte 2008; Ramírez-Sánchez et al., 2011; Pérez-Ramírez et al., 2012; McCay et al., 2014; Álvarez 2014; Álvarez et al., 2015; WinklerPrins 2016).

Ambos casos tienen un precedente en común, la cooperativa fundada en 1930 en Ensenada y que se fragmentó por conflictos internos en 1939 (Estes 1977; García 2009; Ramírez-Sánchez et al., 2011).

Para comprender los esquemas de comanejo en las regiones pesqueras aquí presentadas se utilizó un enfoque metodológico histórico ambiental basado en el conocimiento local (local knowledge) e integrando el conocimiento formal (científico, oficial), como lo muestran estudios recientes (Pawson y Dovers 2003). Esto, con el fin de contrastar, comparar, complementar y resolver la aparente contradicción entre ambos conocimientos (Dahlberg y Blaikie 1999; Dahlberg 2000).

Se conjuntaron una serie de técnicas de investigación de las ciencias naturales, sociales y antropológicas. Asimismo, se incorporaron métodos interdisciplinarios que han sido adoptados en estudios histórico-ambientales, histórico-geográficos y social-ecológicos (Galindo 1998; Taylor y Bogdan 1998; Pawson y Dovers 2003; Andrews y Kearns 2005; Wiles et al., 2005; Andrews et al., 2006; Tjora 2006; Endfield 2009, 226; Martin y Pavlovskaya 2009, 370380; DeLyser et al., 2010; Hay 2010; Memon y Kirk 2011, 107; Belton 2012, 135-136; Lauer 2012).

Los métodos y técnicas empleadas han sido descritos en Álvarez (2014) y Delgado (2014). Para la recopilación de la información empírica, se realizó trabajo de campo en ambos casos, utilizando principalmente el método etnográfico, técnicas de investigación cualitativa y cartografía participativa (cuadro 1). Con la información recabada se realizó un proceso de triangulación en ambos estu- 


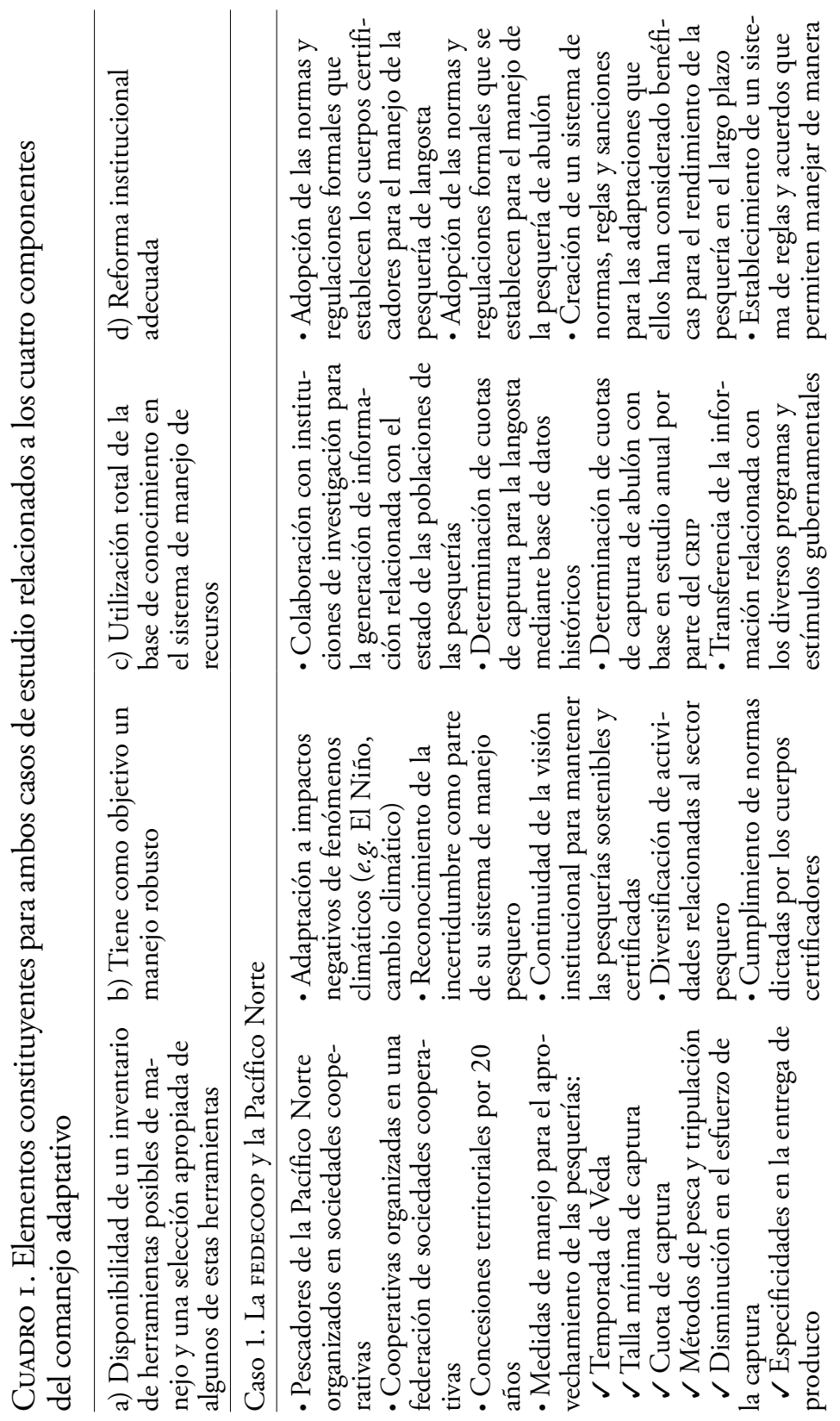




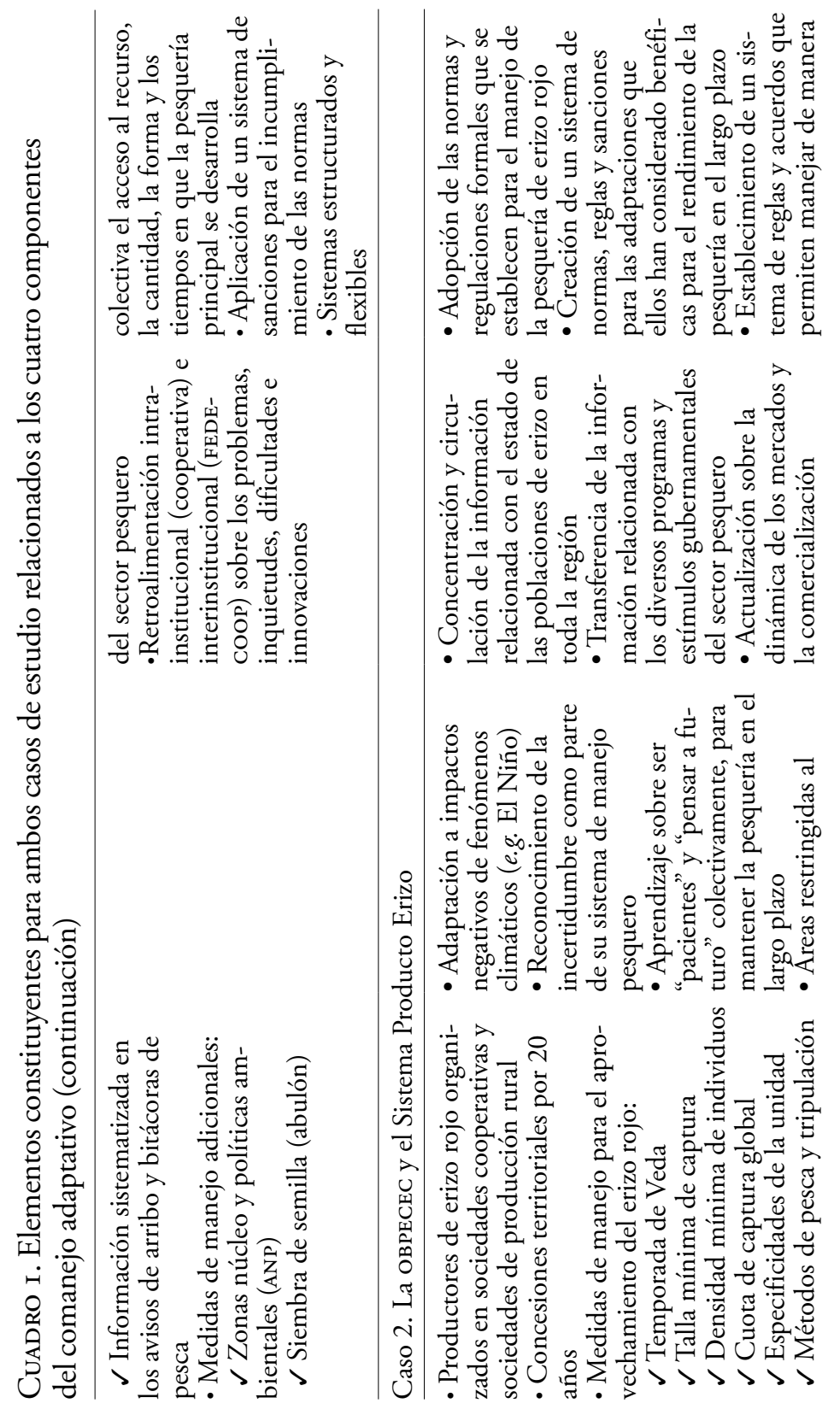



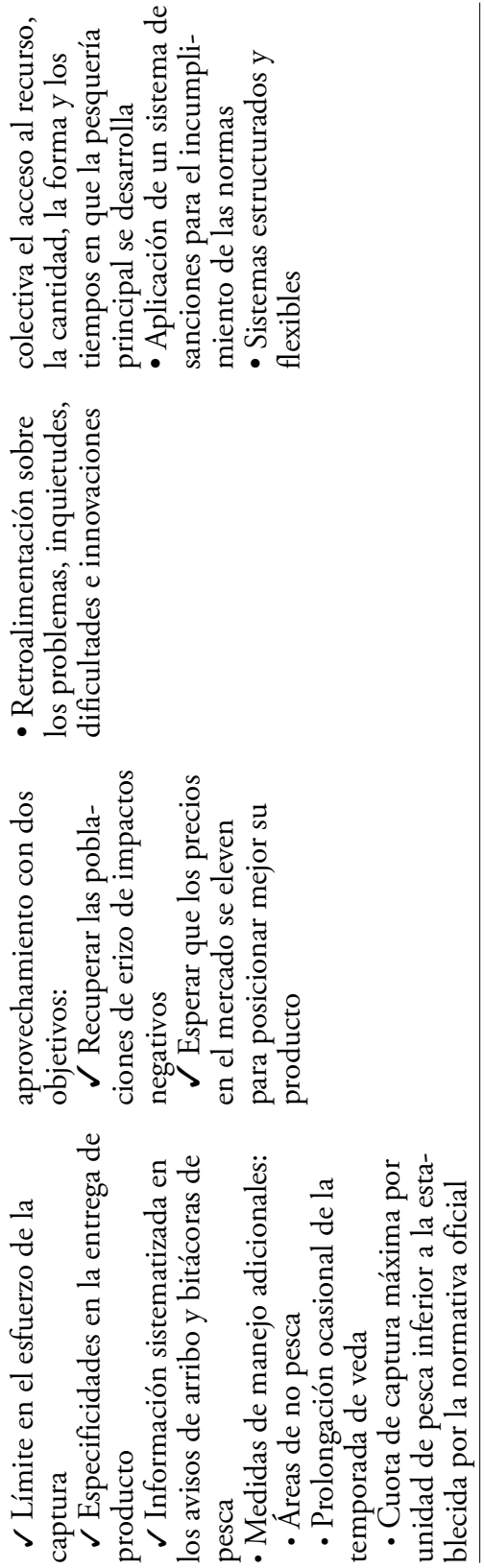
dios de caso para validar los datos y resultados obtenidos (Castillo $e t$ al., 2005; Hay 2010, 77-78), esto permitió resaltar la complejidad y riqueza de la investigación de manera rigurosa (Denzin y Lincoln 2000; Hay 2010, 69-79).

En este sentido, los estudios de caso aquí expuestos representan una oportunidad para entender el diálogo entre las preocupaciones y experiencias del pasado y presente, que incluso incluye el camino para escribir el futuro (O’Connor 1997), como una contribución para encontrar soluciones a la sobreexplotación de las pesquerías, a los conflictos internos de las comunidades pesqueras y la crisis ecológica global de la zona costera en un contexto de cambio climático. Además, con este tipo de estudios se pretende contribuir en la generación de experiencia en las disciplinas sociales en el estudio del desarrollo en materia de pesquerías y acuacultura (Belton 2012, 134).

\section{Resultados}

\section{La FEDECOOP y La Pacifico Norte}

Las comunidades pesqueras que comprenden la región pesquera conocida como La Pacífico Norte están organizadas en cooperativas pesqueras y forman parte de la Federación Regional de Sociedades Cooperativas de la Industria Pesquera Baja California, F.C.L. (FEDECOOP). Estas comunidades están localizadas en la costa central de la Península de Baja California, en el estado de Baja California Sur, municipio de Mulegé. Están limitadas al norte, sur y occidente por el Océano Pacífico, y al oriente por el desierto de El Vizcaíno (figura 1b). El proceso de conformación de estas cooperativas pesqueras tiene tres momentos: a) el cambio de oficio de rancheros a pescadores; b) la formación y consolidación de las organizaciones pesqueras; y c) las concesiones pesqueras de langosta y abulón.

\section{El cambio de oficio de rancheros a pescadores}

El origen de estas comunidades pesqueras se remonta a inicios del siglo XX, cuando una empresa japonesa exploró las costas de Califor- 
nia y el entonces Territorio de Baja California para determinar la viabilidad de la pesquería de abulón. Esta empresa comenzó a explotar dicho recurso en 1912 desde la frontera con Estados Unidos hasta Bahía Magdalena, obteniendo la concesión del gobierno Mexicano postrevolucionario.

En los primeros ańos de operaciones, la empresa japonesa establecía campamentos temporales de marzo a noviembre, transportando lo necesario para el oficio de la pesca vía marítima, incluyendo la mano de obra que era de origen japonés. Sin embargo, comenzó a contratar rancheros de la región, enseñándoles el oficio de la pesca, con el fin de aumentar la cuota de captura basada en un sistema intensivo de manejo de la pesquería.

A principios de 1930, la empresa japonesa se declara en bancarrota, debido a la crisis económica de la Gran Depresión, posterior a la Primera Guerra Mundial, por lo que termina sus operaciones en la región. Como consecuencia, los nuevos pescadores mexicanos decidieron continuar con el oficio de la pesca, estableciendo la primera comunidad pesquera llamada Bahía Tortugas y campos pesqueros permanentes

A partir de entonces comenzó un proceso de apropiación del territorio costero y del ambiente marino por parte de estos pobladores de origen ranchero. Las condiciones ambientales adversas imperantes en la región fueron el principal factor para moldear la dinámica social entre los pescadores y sus familias. Estas condiciones ambientales eran la escasez extrema de agua potable, la ausencia de recursos como madera y material para construcción, las condiciones climáticas desérticas, y la dificultad para transportar bienes y servicios debido al aislamiento geográfico.

La estrategia de subsistencia entre 1930 y 1950 fue la repartición de tareas entre los pescadores y sus familias, mientras unos salían a pescar, otros desalinizaban y racionaban el agua, otros más reunían madera o realizaban la limpieza de las artes de pesca y de los artículos del hogar. Los recursos eran compartidos de manera equitativa según el número de miembros en cada familia. Esta dinámica fue desarrollando una organización social informal pero fuertemente cohesionada y con sentido de cooperación. El cuidado de los recursos que eran 
muy escasos, en especial el agua, fomentó un sentido de responsabilidad y pertenencia comunal, reforzado por lazos familiares estrechos que actualmente prevalecen, ya que las familias eran originarias de las rancherías cercanas y de San Ignacio, Baja California Sur. Además, las pesquerías, al ser la única actividad económica históricamente viable asociada a la costa, han fungido como el eje del desarrollo, permitiendo que se mantengan amalgamadas las comunidades.

\section{La formación y consolidación de las organizaciones pesqueras}

Para finales de 1960, los pescadores se habían constituido en seis cooperativas pesqueras, ${ }^{2}$ formalizando la organización social que prevalecía entre ellos. Además, se fundaron tres comunidades más: Bahía Asunción, La Bocana y Punta Abreojos.

Las cooperativas comenzaron a proveer de los servicios básicos a las comunidades, dotándolas de agua potable y luz eléctrica. Desde mediados de siglo las cooperativas se encargaron de abrir caminos de terracería aptos para la entrada de vehículos, que los comunicaban a la carretera Transpeninsular, construida en la década de 1970. Por esta razón, el transporte de bienes a las comunidades y la exportación de las capturas dejaron de ser vía marítima.

Las cooperativas diversificaron sus pesquerías y hubo un reacomodo de éstas debido principalmente a la demanda del mercado internacional. La pesquería de langosta se posicionó como la primera en total de captura y de ingreso para las cooperativas, seguida por la pesquería de abulón que había disminuido significativamente las cotas de captura. Otras pesquerías que se desarrollaron fueron la de almeja, pepino de mar y escama en general. El esquema de manejo de las pesquerías continuaba siendo intensivo, aunque habían entrado en vigor leyes y vedas decretadas por el gobierno mexicano.

En la época entre 1960 y 1980, la región de La Pacífico Norte no estuvo exenta de los conflictos laborales y sociales alrededor del

${ }^{2}$ Los nombres de las cooperativas fueron: Bahía Tortugas, Emancipación, La Purísima (localidad de Bahía Tortugas); California de San Ignacio (localidad de Bahía Asunción); Progreso (localidad de La Bocana); y Punta Abreojos (localidad de Punta Abreojos). 
mundo. Si bien, los pescadores estaban constituidos como cooperativas, los ingresos y las decisiones habían sido acaparados por los administradores. Esto había sido consecuencia en gran medida porque los pescadores, socios de las cooperativas, no tenían conocimiento sobre el funcionamiento y administración de una cooperativa. Sin embargo, con la llegada de varios líderes sindicales provenientes de la Confederación de Trabajadores de México (Стм) a la región, se comienza con una concientización de los pescadores. A raíz de ello, se desencadena un conflicto, donde eventualmente el gobierno federal interviene, que da como resultado la expulsión de dichos administradores y la entrada de juntas directivas formadas por los mismos socios pescadores, elegidas por ellos mismos y con un recambio periódico.

\section{Las concesiones pesqueras de langosta y abulón}

En la década de 1980, las cooperativas pesqueras observaron una disminución dramática en las cuotas de captura en las pesquerías de langosta y abulón por lo que, con asesoría de técnicos de dependencias de gobierno e investigadores de las universidades de la región, determinaron que estaban sobreexplotadas como consecuencia de un sistema intensivo de pesca que no había cambiado desde inicios de siglo.

La respuesta de las cooperativas fue buscar la ayuda de instituciones de investigación para determinar qué estaba sucediendo, cómo podían recuperar las cuotas de captura y cómo mantener estables las pesquerías. Esta decisión representó una estrategia interna de manejo colaborativo (down-top), resultado de la organización y cohesión social de las cooperativas. Esto representó el inicio de un esquema de comanejo de sus pesquerías.

A inicios de 1990, las cooperativas pesqueras colaboraban con dependencias de gobierno en sus tres niveles, instituciones de investigación, y organizaciones públicas y privadas. ${ }^{3}$ Cada una ha cumpli-

${ }^{3}$ Dentro de las más involucradas están el Centro Regional de Investigación Pesquera (CRIP), Centro de Investigación Biológica del Noreste (CIBNOR), Comunidad y Biodiversidad A.C. (COBI). 
do un papel importante en el esquema de comanejo, como ejemplos, el respeto de las tallas mínimas de captura, apoyos financieros, monitoreo de las pesquerías, cuidado del ambiente y certificaciones, respectivamente. En esta década también sucedió la división de una de las cooperativas, ${ }^{4}$ por lo que en la actualidad existen siete cooperativas pesqueras.

Un primer logro de las cooperativas, como resultado del comanejo fue la obtención de la concesión del acceso exclusivo por 20 ańos para el manejo de sus pesquerías. Otro logro de gran importancia fue la ecocertificación internacional de la pesquería de langosta como pesquería sostenible, ${ }^{5}$ siendo la segunda pesquería de langosta en el mundo con esta distinción, junto con la australiana.

En los últimos años, las cooperativas han adoptado otras estrategias como parte de su proceso adaptativo del comanejo, tales como, una reducción gradual del número de socios pescadores, con el fin de reducir las cuotas de captura; el reemplazo de trampas para langosta tradicionales por biodegradables; y un programa interno de monitoreo estacional de las pesquerías. Recientemente, el gobierno les renovó la concesión por 20 años del acceso exclusivo a sus pesquerías y mantiene la certificación internacional de la pesquería de langosta. Por último, enfrentan dos retos, el primero de ellos, los eventos oceanográficos nocivos y no predecibles, relacionados al cambio climático; y el segundo, la partida de las nuevas generaciones hacia otros estilos de vida, motivado en gran medida por la entrada de los servicios de comunicación que está provocando una apertura de las comunidades pesqueras hacia un mundo globalizado.

\section{La obpecec y el Sistema Producto Erizo}

La organización Buzos y Pescadores del Ejido Coronel Esteban Cantú S.P.R. de R.L. (OBPECEC) se encuentra ubicada en la región noroeste del litoral bajacaliforniano y surge por iniciativa de ejidata-

${ }^{4}$ La cooperativa ubicada en la localidad de Bahía Asunción se dividió en dos cooperativas, la nueva cooperativa lleva por nombre Leyes de Reforma.

${ }^{5}$ Certified sustainable food, Marine Stewardship Council (www.msc.org). 
rios y avecindados que se dedicaban desde los años sesenta a la pesca y buceo ribereńos (figura 1a). El proceso de conformación de esta organización pesquera tiene tres momentos: a) la constitución del ejido Coronel Esteban Cantú; b) la formación y consolidación de la organización pesquera; y c) la concesión pesquera de erizo rojo.

\section{La constitución del ejido Coronel Esteban Cantú}

La conformación del ejido inicia a finales de la década de los sesenta, cuando algunos campesinos dispersos en otros ejidos, se agruparon y solicitaron tierras a la Secretaría de la Reforma Agraria. Para principios de los setenta y por mandato del gobernador del estado, se les otorgó una superficie de 10,119 hectáreas que fueron ejecutadas rápidamente, por el entonces presidente Lic. Luis Echeverría Álvarez. El recién conformado ejido recibió el nombre Ejido Coronel Esteban Cantú, en honor a un gobernador de Baja California. El acta de posesión y deslinde se entregó a los ejidatarios a finales de los ochenta y, a principios de este siglo, los ejidatarios se incorporaron al Programa de Certificación de Tierras Ejidales, obteniendo los certificados parcelarios para asignar y delimitar las tierras ejidales. Actualmente, el ejido tiene 89 ejidatarios con derechos agrarios vigentes.

\section{La formación y consolidación de la organización pesquera}

Algunos de los ejidatarios en Esteban Cantú se dedicaban a la pesca ribereña de escama y al buceo de abulón, langosta y erizo rojo, dedicaban su producción al consumo familiar y a los mercados locales y regionales en Ensenada. No obstante, la falta de documentos que avalaran sus productos era un obstáculo para su comercialización y reducía los precios, razón por la cual decidieron organizar su propia organización cooperativa de pescadores y buzos.

Desde los inicios de la década de los ochenta emprendieron el proyecto de organización cooperativa Aguamarga la cual reunía al grupo de pescadores y buzos libres que trabajaban en la zona de Punta Banda. Este primer proyecto fue fundamental pues fue el 
mecanismo inicial de organización y delimitación interna de espacios de pesca, sin embargo, al no poder obtener permisos de pesca y no poder capitalizarse, los pescadores y buzos decidieron sumarse al ejido del que formaban parte.

A finales de la misma década, el proyecto se convirtió en una Sección Especializada de Producción Ejidal (SEPE) que, si bien, les permitía trabajar de manera regularizada, no les permitía ser autónomos, además, bajo la estructura ejidal se contravenían algunos intereses como contar con personalidad jurídica, tener autoridad en la toma de decisiones y conservar la totalidad del valor de la producción. Debido a esto, cambiaron su figura legal a Sociedad de Producción Rural constituyéndose como tal en el año 1994 con un total de 39 integrantes.

La conformación de la OBPECEC tiene como origen de su capacidad organizativa la propia formación del ejido, pues, la organización pesquera tuvo la capacidad de desarrollar cohesión entre sus miembros y pudo trabajar bajo el aprendizaje de los mismos procesos organizativos y productivos del ejido. Este periodo sienta las bases de una estructura organizativa funcional y adaptativa que posteriormente se replicó al constituirse como SPR.

\section{La concesión pesquera de erizo rojo}

A finales de los años noventa y después de cuatro años de trámites y cabildeo, la organización pesquera recibió una concesión para el aprovechamiento del erizo rojo. El otorgamiento de la concesión no fue casual, obedeció a los intereses económicos y políticos de un empresario que quería una concesión para la engorda de atún en la zona de Punta Banda y a la negociación de la organización pesquera sobre la concesión del erizo rojo a cambio del "permiso" a la empresa atunera para trabajar dentro de su territorio de pesca. Los miembros de la OBPECEC también negociaron con la empresa, que en las contrataciones temporales se diera prioridad a la incorporación de sus buzos y pescadores, acuerdo que sigue vigente hasta la fecha.

La concesión para el aprovechamiento del erizo rojo detonó el diseńo institucional interno, cuya efectividad puede explicarse a la luz 
del aprendizaje social constituido a través de la experiencia institucional ejidal y, posteriormente, en su propia estructura organizativa.

Los miembros de la OBPECEC han acrecentado su capital social e institucional a través de una red de instituciones formales y consuetudinarias. Forman parte de una Asociación de Productores de Erizo de Baja California y a través de ésta participan de manera activa en el Sistema Producto Erizo ${ }^{6}$ (SPE). Además, algunos de los integrantes de las mesas directivas que han tenido, han colaborado con investigadores del CRIP y mantienen una relación con las autoridades pesqueras del estado. Esto les ha permitido establecer una serie de alianzas que redundan en beneficios para el manejo de los recursos pesqueros y en una mayor fluidez de la comunicación con estas autoridades gubernamentales, logrando así reducir los costos de transacción.

En las reuniones del SPE se socializan diferentes tipos de información, por ejemplo, las instancias gubernamentales informan sobre los programas y apoyos que dará el gobierno y los periodos para tramitar los apoyos económicos y algunas instancias académicas llevan información relacionada con el estado de la pesquería. También se hace un seguimiento de actividades como los avances en el programa de trasplantes de erizo rojo y cada uno de los miembros muestra sus propuestas, sus experiencias y los problemas relacionados con la pesquería tales como la pesca ilegal o la sobreexplotación de sargazo (Macrocystis pyrifera) y su impacto en diversas pesquerías. El SPE es un espacio de relaciones respetuosas y colaborativas, aun cuando la composición del grupo es heterogénea, tienen como objetivo común la sustentabilidad de la pesquería. Con base en el análisis anterior, la colaboración entre usuarios, comunidades y gobierno, es decir, el esquema de comanejo, está desarrollando fuertes lazos y alianzas entre los productores y las instituciones gubernamentales. En este sentido, el comité es efectivo como un potenciador del capital social.

${ }^{6}$ Los sistemas de productos y sus comités han sido promovidos por SAGARPA para fortalecer y consolidar cadenas productivas mediante la formación de capital social entre los sectores productivos. 


\section{Comanejo adaptativo en La Pacífico Norte y el Sistema Producto Erizo}

Se observa como ambos casos tuvieron caminos diferentes en su desarrollo como comunidades pesqueras y organizaciones sociales que alcanzaron un destino en común: el comanejo adaptativo. Si bien, cada proceso histórico de desarrollo ha sido particular, se encontraron momentos similares por los que pasaron estas comunidades pesqueras para alcanzar el comanejo adaptativo (figura 2).

En ambos casos se observa un cambio de oficio, trasladando elementos organizativos de sus anteriores actividades. En el caso de $\mathrm{La}$ Pacífico Norte la cultura del ranchero y las condiciones ambientales adversas fueron los factores que propiciaron la organización en cooperativas pesqueras. Para el Sistema Producto Erizo, la organización que tenían previamente establecida como ejidatarios fue principalmente lo que permitió que se constituyera la cooperativa pesquera. Se observa como en ambos casos estas organizaciones pesqueras; que desde un inicio estuvieron cimentadas con pilares como la responsabilidad compartida, el sentido de pertenencia comunal por el recurso y la repartición de tareas, fueron tomando decisiones internas y resolviendo problemáticas que las llevó de un sistema intensivo de manejo de sus pesquerías a un comanejo de ellas, y recién a un

Figura 2. Proceso de desarrollo en el manejo de las pesquerías de La Pacífico Norte y el Sistema Producto Erizo

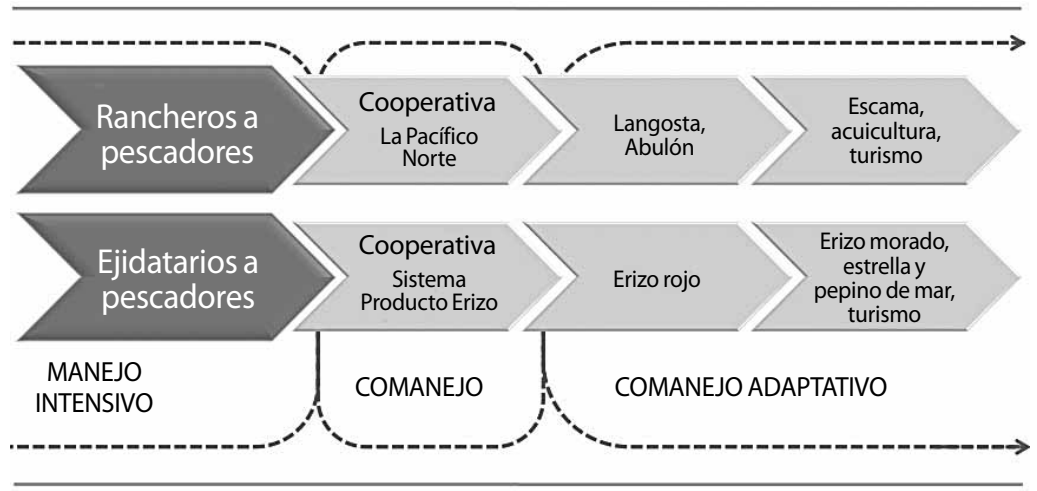


comanejo adaptativo, el cual les ha permitido diversificar sus pesquerías y, actualmente, empezar a encontrar actividades alternas como la acuicultura y ecoturismo; así como enfrentar problemáticas nuevas como el cambio climático, impactos ambientales adversos, migración y un mundo globalizados.

\section{Conclusiones}

La Pacifico Norte y el Sistema Producto Erizo cristalizan el comanejo adaptativo en sus dos aspectos centrales. Primero, el comanejo, pues, convergen los diversos niveles de gobierno, tales como el municipal, el estatal y el federal (vertical); los diversos usuarios de la pesquería del erizo de mar, como los miembros de organizaciones del sector social (cooperativas o sociedades de producción rural), productores privados (permisionarios) y comercializadores; así como organizaciones de la sociedad civil e instituciones de investigación. Segundo, el aprendizaje continuo, dado que en La Pacífico Norte las zonas de pesca, las cuotas de captura, las artes de pesca, son ejemplos de la integración de los conocimientos local y científico, mientras que en el Sistema Producto Erizo el sistema de trasplantes de erizo, que actualmente se lleva a cabo en diversas comunidades pesqueras, ha resultado de la combinación del conocimiento ecológico local de los buzos y pescadores con el conocimiento científico de diversos especialistas. Para explicar el proceso que llevó a estas comunidades a construir dicho esquema de manejo se utilizó un enfoque histórico ambiental, el cual sirve de guía para revelar las interacciones entre los sistemas social y natural que han moldeado el desarrollo, manejo y toma de decisiones sobre sus pesquerías. También permite la identificación de las posibilidades y modalidades de interacciones entre ambos sistemas, así como llevar a cabo un análisis contextual para identificar fuerzas internas y externas que expliquen los cambios en la historia de estas comunidades pesqueras. En los dos casos, el grado de organización interna que alcanzaron estas comunidades pesqueras ha sido el resultado de un proceso en el que se sucedió una serie de condiciones particulares que permitieron establecer el comanejo adaptativo. Si bien, son difícilmente replica- 
bles, ya que son procesos singulares de cada comunidad pesquera, pueden ser la premisa para buscar, en otros casos, condiciones particulares presentes y utilizarlas como fortalezas y áreas de oportunidades para establecer esquemas sostenibles de manejo de recursos.

\section{Agradecimientos}

Los autores del presente artículo agradecemos al Dr. Gerardo Bocco, Dr. Hugo Riemann y la Dra. Micheline Cariño por su valioso apoyo en el análisis y colaboración en las tesis doctorales que fueron la base para el presente texto. Además, queremos extender un especial agradecimiento al Dr. Gerardo Bocco por el apoyo para la realización del trabajo de campo. Por último, un sincero agradecimiento a los revisores de la revista Relaciones Estudios de Historia y Sociedad y a los coordinadores de la sección temática "Pesquerías, política pesquera, desarrollo y conservación en el Noroeste de México".

La investigación origen del presente texto fue patrocinada por los proyectos Cultural Landscapes of Coastal Baja California Sur: Stability Amidst Change, National Geographic Society 9186-12; DGAPA-PAPIIT, UNAM IN305010; Pequeñas localidades y redes de estructuración territorial en lo remoto y La construcción social del paisaje en pequeñas localidades rurales en Baja California (México) y La Patagonia Atlántica (Argentina), CONACYT y Ministerio de Ciencia (Argentina); UABC-INECC-PNUD 868487 de la Plataforma de Colaboración sobre Cambio Climático y Crecimiento Verde entre Canadá y México 2013-2016; tesis doctoral "Desarrollo, cultura y manejo de recursos pesquero-ribereños en el Pacífico de Baja California. El papel de las instituciones y el conocimiento ecológico local en la organización Buzos y Pescadores del Ejido Coronel Esteban Cantú". CONACYT, El Colegio de la Frontera Norte A.C.

\section{REFERENCIAS}

Álvarez, P. M. 2014. "Construcción del paisaje cultural en las comunidades pesqueras del Pacifico Norte Mexicano". Tesis Doctoral, Medio Ambiente y Desarrollo, UABC. 
Álvarez, P. M., G. Seingier, I. Espejel, G. Bocco y J. Noriega. 2015. "Evolution Of The Regional Landscape of Fishing Communities on the Pacific Coast of Northern Mexico. Landscape Research 40(7) (2015): 855-874.

Andrews, G. J. y R. A. Kearns. 2005. Everyday Health Histories and the Making of Place: The Case of an English Coastal Town. Social Science \& Medicine 60(12): 2697-2713.

Andrews, G. J., R. A. Kearns, P. Kontos y V. Wilson. 2006. "'Their Finest Hour': Older People, Oral Histories, and the Historical Geography of Social Life", Social \& Cultural Geography 7(2): 153-177.

Arce, R. y F. Sotero. 2015. Punta Abreojos 1948-2005. Punta Abreojos: S.C.P.P. "Punta Abreojos" S.C.L. Punta Abreojos, Baja California Sur, 107 pp.

Armitage, D., F. Berkes y N. Doubledays, eds. 2007. Adaptive Co-management. Collaboration, Learning, and Multiple Level Governance. Vancouver: UBC Press.

Barton, J. R. 2006. "Sustainable Fisheries Management in the Resource Periphery: The Cases of Chile and New Zealand". Asia Pacific Viewpoint 47(3): 366-380.

Belton, B. 2012. "Culture, Social Relations and Private Sector Development in the Thai and Vietnamese Fish Hatchery Sectors". Asia Pacific Viewpoint 53(2): 133-146.

Bourassa, S. C. y A. L. Strong. 2000. "Restitution of Fishing Rights to Maori: Representation, Social Justice and Community Development". Asia Pacific Viewpoint 41 (2): 155-175.

Butzer, K. W. 1971. Environment and Archeology: An Ecological Approach to Prehistory. Chicago: Aidine-Atherton.

Carey, M. 2009. "Latin American Environmental History: Current Trends, Interdisciplinary Insights, and Future Directions". Environmental History 14(2): 221-252.

Cariño, M. y M. Monteforte. 2008. Del saqueo a la conservación: Historia ambiental contemporánea de Baja California Sur, 19402003. México: Secretaría de Medio Ambiente y Recursos Naturales, Instituto Nacional de Ecología, Universidad Autónoma de Baja California Sur, Consejo Nacional de Ciencia y Tecnología. 
Carr, L. M., y W. D. Heyman. 2012. “It's About Seeing What's Actually Out There”: Quantifying Fishers' Ecological Knowledge and Biases in a Small-Scale Commercial Fishery as a Path Toward Co-management. Ocean \& Coastal Management (69): 118-132.

Castillo, A., A. Torres, A. Velázquez y G. Bocco. 2005. “The Use of Ecological Science by Rural Producers: A Case Study in Mexico". Ecological Applications 15(2): 745-756.

Castree, N., D. Demeritt y D. Liverman. 2009. "Introduction: Making Sense of Environmental Geography”. En A Companion to Environmental Geography, ed. N. Castree, D. Liverman y B. Rhoads. Singapur: Wiley-Blackwell.

Castro, H. G. 2002. "Naturaleza, sociedad e historia en América Latina". En Ecología Politica. Naturaleza, sociedad y utopia, ed. H. Alimonda. Buenos Aires: CLACSO.

. 2010. "La Historia Ambiental latinoamericana. Recuentos y disyuntivas". Paper presented at the Simposio Internacional "Cultura, ciencia y naturaleza. Actualidad del pensamiento de Antonio Núñez Jiménez”.

Charles, Anthony. 2007. "Adaptive Co-management for Resilient Resource Systems: Some Ingredients and the Implications for their Absence". En Adaptive Co-management: Collaboration, Learning and Multi-level Governance, ed. Derek Armitage, Fikret Berkes y Nancy Doubleday, 83-102. Vancouver: UBC press.

Dahlberg, A. C. 2000. "Landscape(s) in Transition: An Environmental History of a Village in North-east Botswana". Journal of Southern African Studies 26(4): 759-782.

Dahlberg, A. C., y P. M. Blaikie. 1999. "Changes in Landscape or in Interpretation? Reflections Based on the Environmental and Socio-economic History of a Village in NE Botswana”. Environment and History 5(2): 127-174.

Dedina, S. L. 1996. The Production of Gray Whale Conservation in Baja California Sur, Mexico. Austin: Texas University.

Delgado, C. 2014. "Desarrollo, cultura y manejo de recursos pesquero-ribereños en El Pacífico de Baja California. El papel de las instituciones y el conocimiento ecológico local en la organi- 
zación Buzos y Pescadores del Ejido Coronel Esteban Cantú". Tesis Doctorado, Colef, 258 pp.

DeLyser, D., S. Herbert, S. Aitken, M. Crang y L. McDowell. 2010. The Sage Handbook of Qualitative Geography. Thousand Oaks: Sage Publications.

Denzin, N. K. y Y. S. Lincoln. 2000. Handbook of Qualitative Research. Thousand Oaks: Sage Publications.

Díaz-Uribe, J. G., V. M. Valdez-Ornelas, G. D. Danemann, E. Torreblanca-Ramírez, A. Castillo-López y M. A. CisneROS-MATA. 2013. "Regionalización de la pesca ribereña en el noroeste de México como base práctica para su manejo". Ciencia Pesquera 21(1): 41-54.

Edwards, V. M. y N. A. Steins. 1999. A Framework for Analyzing Contextual Factors in Common Pool Resource Research. Journal of Environmental Policy and Planning 1(3): 205-221.

Endfield, G. H. 2009. "Environmental History". En A Companion to Environmental Geography, ed. N. Castree, D. Demeritt, D. Liverman y B. Rhoads. Singapur: Wiley-Blackwell.

Espinoza-Tenorio, A., I. Espejel, M. Wolff y J. A. Zepeda-DoMínguez. 2010. Contextual Factors Influencing Sustainable Fisheries in Mexico. Marine Policy 35(3): 343-350.

Espinoza-Tenorio, A., I. Espejel, y M. Wolff. 2015. "From Adoption to Implementation? An Academic Perspective on Sustainable Fisheries Management in a Developing Country". Marine Policy 62(C): 252-260.

Estes, H. D. 1977. "Kondo Masaharu and the Best of All Fisherman". The Journal of San Diego History 23(3). http://www.sandiegohistory.org/journal/1977/july/kondo/ (Fecha de consulta: 8 de agosto de 2011).

Fernández-Boán, M., L. Fernández y J. Freire. 2012. "History and Management Strategies of the Sea Urchin Paracentrotus Lividus Fishery In Galicia (NW Spain)". Ocean \& Coastal Management, vol. 69 (diciembre de 2012): 265-272.

Fulton, E. A., A. D. Smith, D. C. Smith y I. E. Van Putten. 2011. Human Behaviour: the Key Source of Uncertainty in Fisheries Management. Fish and Fisheries 12(1): 2-17. 
Galindo, C. L. J. 1998. Técnicas de investigación en sociedad, cultura y comunicación. México: Pearson Educación.

Gallini, S. 2009. "Historia, ambiente, política: el camino de la historia ambiental en América Latina", Nómadas (30): 92-102.

García, C. A. 2009. Bahia Tortugas Monografía. Baja California Sur. México: Compás.

Hay, I. 2010. Qualitative Research Methods in Human Geography. Nueva York: Oxford University Press.

Hornborg, A. 2007. "Introduction: Environmental History as Political Ecology”. En Rethinking Environmental History: WorldSystem History and Global Environmental Change, ed. A. Hornborg, J. R. McNeill y J. M. Alier. Lanham: Altamira Press. Hughes, J. D. 2008. “Three Dimensions of Environmental History". Environment and History 14(3): 319-330.

Jorgenson, A. K. y J. Rice. 2007. "Uneven Ecological Exchange and Consumption-Based Environmental Impacts: A Cross-National Investigation". En Rethinking Environmental History: World-System History and Global Environmental Change, ed. A. Hornborg, J. R. McNeill y J. M. Alier. Lanham: Altamira Press. Jentoft, S. 1989. "Fisheries Co-management: Delegating Government Responsibility to Fishermen's Organizations". Marine policy 13(2): 137-154.

Jentoft, S. y B. McCay. 1995. "User Participation In Fisheries Management: Lessons Drawn from International Experiences". Marine Policy 19(3): 227-246.

Jentoft, S. 2000a. “The Community: A Missing Link of Fisheries Management". Marine Policy 24(1): 53-60.

Jentoft, S. 2000b. "Legitimacy and Disappointment In Fisheries Management". Marine Policy 24(2): 141-148.

Lauer, M. 2012. "Oral Traditions or Situated Practices? Understanding How Indigenous Communities Respond to Environmental Disasters". Human Organization 71(2): 176-187.

Martin, K. S. y M. Pavlovskaya. 2009. "Ethnography”. En $A$ Companion to Environmental Geography, ed. N. Castree, D. Demeritt, D. Liverman y B. Rhoads. Singapur: Wiley-Blackwell. 
McCay, B. J., F. Micheli, G. Ponce-Díaz, G. Murray, G. Shester, S. Ramirez-Sanchez y W. Weisman. 2014. "Cooperatives, Concessions, And Co-Management On The Pacific Coast Of Mexico". Marine Policy 44(C): 49-59.

McNeILl, J. R. 2000. Something New Under the Sun: An Environmental History of the Twentieth-Century World. Nueva York: Norton.

Memon, P. A. y N. A. Kirk. 2011. "Maori Commercial Fisheries Governance in Aotearoa/New Zealand within the Bounds of a Neoliberal Fisheries Management Regime". Asia Pacific Viewpoint 52(1): 106-118.

Miller, S. W. 2007. An Environmental History of Latin America. Nueva York: Cambridge University Press.

Muawanah, U., R. S. Pomeroy y C. Marlessy. 2012. "Revisiting Fish Wars: Conflict and Collaboration over Fisheries in Indonesia”. Coastal Management 40(3): 279-288.

O'Connor, J. 1997. "What is Environmental History? Why Environmental History?”, Capitalism Nature Socialism 8(2): 3-29.

Olsson, P., C. Folke y T. P. Hughes. 2008. "Navigating the Transition to Ecosystem-Based Management of the Great Barrier Reef, Australia”. Proceedings of the National Academy of Sciences 105(28), 9489-9494.

Pawson, E., y S. Dovers. 2003. "Environmental History and the Challenges of Interdisciplinarity: An Antipodean Perspective", Environment and History 9(1): 53-75.

Pérez-Ramírez, M., G. Ponce-Díaz y S. Lluch-Cota. 2012. "The Role of MSC Certification in the Empowerment of Fishing Cooperatives in Mexico: The Case of Red Rock Lobster Co-managed Fishery". Ocean \& Coastal Management, vol. 63 (julio 2012): 24-29.

Pinkerton, E. 2007. "Integrating Holism and Segmentalism: Overcoming Barriers to Adaptive Co-management between Management Agencies and Multi-Sector Bodies". En Adaptive Co-management: Collaboration, Learning and Multi-level Governance, ed. Derek Armitage, Fikret Berkes y Nancy Doubleday, 151-171. Vancouver: UBC press.

Ponce-Díaz, G., A. Vega-Velázquez, M. Ramade-Villanueva, 
G. León-Carballo y R. Franco-Santiago. 1998. "Socioeconomic Characteristics Of The Abalone Fishery along the West Coast of the Baja California Peninsula, Mexico". Journal of Shellfish Research 17(3): 853-857.

Plummer, Ryan y John FitzGibbon. 2007. "Connecting Adaptive Co-management, Social Learning and Social Capital Through Theory and Practice". En Adaptive Co-management: Collaboration, Learning and Multi-level Governance, ed. Derek Armitage, Fikret Berkes y Nancy Doubleday, 38-61. Vancouver: UBC press.

Ramírez Sánchez, S., B. J. McCay, T. R. Johnson y W. WeisMAN. 2011. "Surgimiento, formación y persistencia de organizaciones sociales para la pesca ribereña de la península de Baja California". Región y Sociedad 23(51): 71-99.

Roberts, C. 2009. The Unnatural History of the Sea. Londres: Island Press.

Rucinque, H. F. y A. L. Velásquez. 2007. “Geografía e historia: ¿Reactivación de antiguas relaciones interdisciplinarias?”, Revista de Relaciones Internacionales, Estrategia y Seguridad 2(2): 127148.

Sáenz-Arroyo, A., C. M. Roberts, J. Torre, M. Cariño-Olvera y R. R. EnríqueZ-Andrade. 2005. "Rapidly Shifting Environmental Baselines among Fishers of the Gulf of California”, Proceedings of the Royal Society of London B: Biological Sciences 272(1575): 1957-1962.

Sauer, C. O. 1938. Destructive Exploitation in Modern Colonial Expansion. Comptes Rendus du Congrès International de Géographie, Amsterdam 2(3c): 494-499.

. 2005. La gestión del hombre en la tierra y otros ensayos. Selección, traducción y presentación de Guillermo Castro H. Panamá: Ed. Ciudad del Saber.

TAYLOR, S. y R. Bogdan. 1998. Introducción a los métodos cualitativos de investigación. México: Paidós.

TJora, A. H. 2006. "Writing Small Discoveries: An Exploration of Fresh Observers' Observations”. Qualitative Research 6(4): 429451. 
Weisz, H. 2007. "Combining Social Metabolism and InputOutput Analyses to Account for Ecologically Unequal Trade". En Rethinking Environmental History: World-System History and Global Environmental Change, ed. A. Hornborg, J. R. McNeill y J. M. Alier. Lanham: Altamira Press.

Wiles, J. L., M. W. Rosenberg y R. A. Kearns. 2005. "Narrative Analysis as a Strategy for Understanding Interview Talk in Geographic Research". Area 37(1): 89-99.

Wilk, R. 2007. "The Extractive Economy: An Early Phase of the Globalization of Diet, and its Environmental Consequences". En Rethinking Environmental History: World-System History and Global Environmental Change, ed. A. Hornborg, J. R. McNeill y J. M. Alier. Lanham: Altamira Press.

WinklerPrins, A., P. Alvarez, G. Bocco e I. Espejel. 2016. "From Ranching to Fishing- The Cultural Landscape of the Northern Pacific Coast of Baja California, Mexico". Focus on Geography https://focusongeography.org/publications/articles/ baja/index.html 